\title{
Screening Electric Fields using a Tube of Water: The Transition from Conductive to Dielectric Screening
}

\author{
J.S. Bobowski and Jaklyn De Vosil \\ Department of Physics, University of British Columbia Okanagan, \\ 3333 University Way, Kelowna, BC, V1V 1V7, Canada
}

(Dated: August 24, 2015)

\begin{abstract}
An experiment that investigates the transition from conductive to dielectric screening of electric fields by a tube of water has been designed for senior physics undergraduates. A parallelplate capacitor is used to generate a uniform electric field. Two concentric acrylic plexiglass tubes pass perpendicularly through the electric field generated between the plates. The region between the tubes can be filled with air or water. An electrode, suspended within the inner plexiglass tube, is used to sense the electric potential at its location. The sensor is designed so that it can be rotated to measure the potential at a second symmetric position. From the difference in the two potentials, the frequency dependence of the magnitude and phase of the electric field can be determined. With deionized water between the tubes, the magnitude and phase of the interior electric field was measured from $100 \mathrm{~Hz}$ to $300 \mathrm{kHz}$. The high-pass filter frequency response expected for a dielectric tube with non-negligible conductivity was observed. Fits to the data yielded a very reasonable experimental value for the ratio of the water's conductivity to its dielectric constant.
\end{abstract}

Keywords: Electromagnetics, Screening, Dielectric, Conductivity, Voltage sensor, Lock-in detection PACS numbers: 01.50.Pa, 07.50.Hp, 77.22.-d

\section{INTRODUCTION}

It is common and convenient to use a conducting enclosure, or Faraday cage, to exclude external electric fields from a region of space. In a Faraday cage, the conduction electrons redistribute in response to the applied field such that they generate an electric field inside the cage that exactly cancels the applied field. It is, however, also possible to attenuate external electric fields using dielectric enclosures. In the case of a dielectric shield, polar molecules within the dielectric material reorient in response to the applied field. This alignment of electric dipoles generates an electric field that aligns with the applied electric field within the dielectric material and partially cancels the applied field inside the enclosure $\mathbf{D}^{\mathbb{\square} \boldsymbol{\square}}$.

Faraday cages and dielectric enclosures can both be used to suppress static electric fields. Oscillating electromagnetic (EM) fields are also effectively screened by Faraday cages provided that any holes in the cage are much smaller than the wavelength of the incident EM radiation and that the cage walls are much thicker than the frequency-dependent EM skin depth. Dielectric enclosures will also suppress EM radiation provided that, at the frequency of the radiation, $\left|\varepsilon_{\mathrm{r}}(\omega)\right| \gg 1$ where $\varepsilon_{\mathrm{r}}(\omega)$ is the frequency-dependent complex relative permittivity of the shield $\mathrm{d}^{\mathrm{D}}$.

In this paper, an experiment designed to study the transition from conductive to dielectric screening by a tube of water is described. This experiment is suitable for senior undergraduate physics students that have completed a course in introductory electrodynamics.

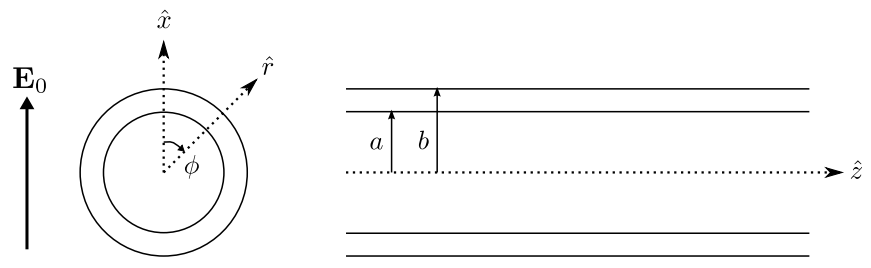

FIG. 1. A dielectric tube of inner radius $a$ and outer radius $b$ immersed in a uniform electric field $\mathbf{E}_{0}$ applied perpendicularly to its axis. Cross-section view (left) and side view (right).

\section{THEORY}

At the interface of two different uncharged dielectric media, Maxwell's equations can be used to show that the perpendicular and parallel components of the electric field $\mathbf{E}$ must satisfy the boundary conditions:

$$
\begin{aligned}
\varepsilon_{1} E_{1_{\perp}} & =\varepsilon_{2} E_{2_{\perp}} \\
E_{1_{\|}} & =E_{2_{\|}}
\end{aligned}
$$

where $\varepsilon_{1}$ and $\varepsilon_{2}$ are the dielectric constants of the two media ${ }^{\boldsymbol{D}}$. The geometry of interest in this experiment is shown in Fig. $\mathbf{⿴}$. A dielectric tube is immersed in a uniform electric field applied perpendicularly to its axis. In this case, the boundary conditions in terms of the electric potential $V$ become:

$$
\begin{aligned}
\varepsilon_{1} \frac{\partial V_{1}}{\partial r} & =\varepsilon_{2} \frac{\partial V_{2}}{\partial r} \\
V_{1} & =V_{2}
\end{aligned}
$$

One way to determine the electric potential in all three regions $(r<a, a<r<b$, and $r>b)$ is to use separation of variables to solve Laplace's equation $\nabla^{2} V(r, \phi)=0$ 
in cylindrical coordinates. Then, one must apply the boundary conditions given by Eqs. $\mathbf{\Xi}$ and $\boldsymbol{\nabla}$ at $r=a$ and $b^{\mathbb{m}, 0}$. The electric field inside the dielectric tube can then be found using $\mathbf{E}=-\nabla V$. The result for a tube of relative permittivity $\varepsilon_{\mathrm{r}}$ immersed in air is:

$$
\mathbf{E}_{\mathrm{in}}=\frac{4 \varepsilon_{\mathrm{r}} \mathbf{E}_{0}}{\left(\varepsilon_{\mathrm{r}}+1\right)^{2}-\left(\frac{a}{b}\right)^{2}\left(\varepsilon_{\mathrm{r}}-1\right)^{2}}
$$

and the shielding effectiveness is defined to be $S E=E_{0} / E_{\text {in }}$ [, . The experiment discussed in the next section investigates the shielding effectiveness of a tube of water. The dielectric constant of room-temperature water is $79 \pm 1$ such that $\varepsilon_{\mathrm{r}} \gg 1$ and $^{\mathbb{\theta}}$ :

$$
S E \approx\left[1-\left(\frac{a}{b}\right)^{2}\right] \frac{\varepsilon_{\mathrm{r}}}{4} .
$$

All water contains a finite concentration of dissolved ions and therefore has a finite conductivity $\sigma$. The charge flow through a cross-sectional area $A$ and length $\ell$ of water can be modelled as parallel conduction through capacitive and resistive impedances:

$$
\begin{aligned}
\frac{1}{Z_{\mathrm{eff}}} & =\frac{1}{Z_{C}}+\frac{1}{Z_{R}}=j \omega \varepsilon_{0} \varepsilon_{\mathrm{r}} \frac{A}{\ell}+\sigma \frac{A}{\ell} \\
& =j \omega \varepsilon_{0}\left(\varepsilon_{\mathrm{r}}-j \frac{\sigma}{\omega \varepsilon_{0}}\right) \frac{A}{\ell}
\end{aligned}
$$

which allows one to characterize the charge dynamics using a capacitive impedance $Z_{\text {eff }}$ with an effective complex and frequency-dependent dielectric constant ${ }^{\mathbf{Q}}$ :

$$
\tilde{\varepsilon}_{\text {eff }}(\omega) \equiv \varepsilon_{\mathrm{r}}\left(1-j \frac{\sigma}{\omega \varepsilon_{0} \varepsilon_{\mathrm{r}}}\right) .
$$

The shielding effectiveness of a tube of water is given by Eq. w with $\varepsilon_{\mathrm{r}}$ replaced by $\tilde{\varepsilon}_{\text {eff }}(\omega)$ such that:

$$
\begin{aligned}
|S E(\omega)|^{-1} & \approx \frac{4 / \varepsilon_{\mathrm{r}}}{1-\left(\frac{a}{b}\right)^{2}} \frac{1}{\sqrt{1+\left(\frac{\sigma}{\omega \varepsilon_{0} \varepsilon_{\mathrm{r}}}\right)^{2}}} \\
\tan \beta & \approx \frac{\sigma}{\omega \varepsilon_{0} \varepsilon_{\mathrm{r}}}
\end{aligned}
$$

where $\beta$ is the phase difference between the applied electric field $\mathbf{E}_{0}$ and the field inside the shield $\mathbf{E}_{\text {in }}$. Note that $|S E(\omega)|^{-1}$ has the frequency dependence of a highpass filter. At high frequencies $\omega \gg \sigma /\left(\varepsilon_{0} \varepsilon_{\mathrm{r}}\right)$, the shielding effectiveness reduces to the form given in Eq. 6 and the screening is determined by the dielectric properties of the water. On the other hand, at low frequencies $\omega \ll \sigma /\left(\varepsilon_{0} \varepsilon_{\mathrm{r}}\right),|S E(\omega)|^{-1} \propto \omega \varepsilon_{0} \varepsilon_{\mathrm{r}} / \sigma$ and goes to zero at $\mathrm{dc}$ as would be expected for a Faraday cage.

Finally, as will be discussed in the following section, the tube of water used in the experiment is formed by filling the space between two concentric plexiglass tubes. The presence of the plexiglass modifies the geometry shown in Fig. $\mathbb{\square}$ as now five regions and matching conditions at four boundaries must be considered (air-plexiglass-waterplexiglass-air). The method used to calculate the shielding effectiveness of this geometry is exactly as described above but the solution is very complicated ${ }^{\mathbf{\Xi}}$. However, $\tan \beta$ and the frequency dependence of the $|S E|^{-1}$ remain unchanged provided that the dielectric constant of plexiglass $(\approx 3.4)$ is much less than that of water $(\approx 80)$.

\section{EXPERIMENTAL DESIGN}

Figure $\nabla$ shows a schematic of the experimental apparatus and Fig. B shows a photograph of the actual apparatus built in-house ${ }^{\mathbf{g}}$. A pair of $1 / 2$ in.-thick aluminum plates (16 in. $\times 16$ in.) separated by 6 in. were used to construct a parallel-plate capacitor that creates uniform electric field. The plates were driven by a center-tapped transformer so as to establish a ground plane midway between the capacitor plates. This allowed a grounded copper tube to extend partially into the region between the plates without resulting in a substantial redistribution of the electric field lines.

The 5/8 in. copper tube supports an electrode sensor and houses a buffer circuit. The sensor was made from a rectangular piece of 0.01 in.-thick of aluminum foil $3.6 \mathrm{in}$. long and 0.1875 in. wide. With the electrode placed to one side of the ground plane, it acquires a voltage that is proportional to the capacitor plate voltage and is dependent on the input impedance of the buffer circuit ${ }^{\text {m. }}$. A simple circuit model that can be used to estimate the voltage acquired by the suspended electrode is shown in Fig. $\boldsymbol{\theta}$. The capacitance $C_{0}^{\prime}$ represents the net capacitance between the electrode and the ground plane which must include the input capacitance of the buffer circuit. The voltage at the buffer output is given by

$$
V_{0}=V \frac{C_{1}-C_{2}}{C_{0}^{\prime}+C_{1}+C_{2}} .
$$

To ensure that the largest possible buffer output is measured, it is important to minimize $C_{0}^{\prime}$ and hence the buffer input capacitance. The op-amp used was an Analog Devices AD549L which has a nominal input capacitance of $0.8 \mathrm{pF}$ (and input resistance $10^{15} \Omega$ ) portant to consider the layout of the buffer circuit so as to minimize stray capacitance contributions to $C_{0}^{\prime}$ that

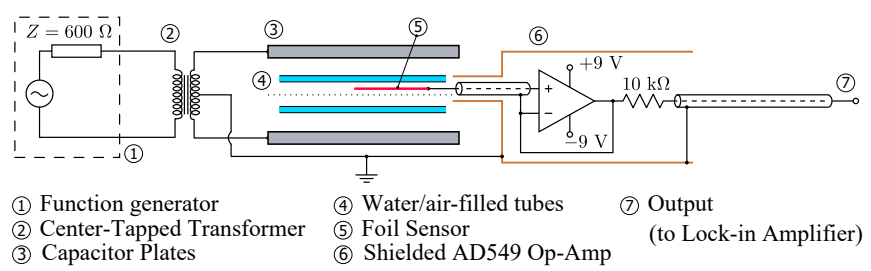

FIG. 2. A parallel-plate capacitor generates a uniform electric field that is perpendicular to the axis of a tube of water. An electrode, used to sense the electric potential, is suspended within the tube of water and feeds into a buffer. 


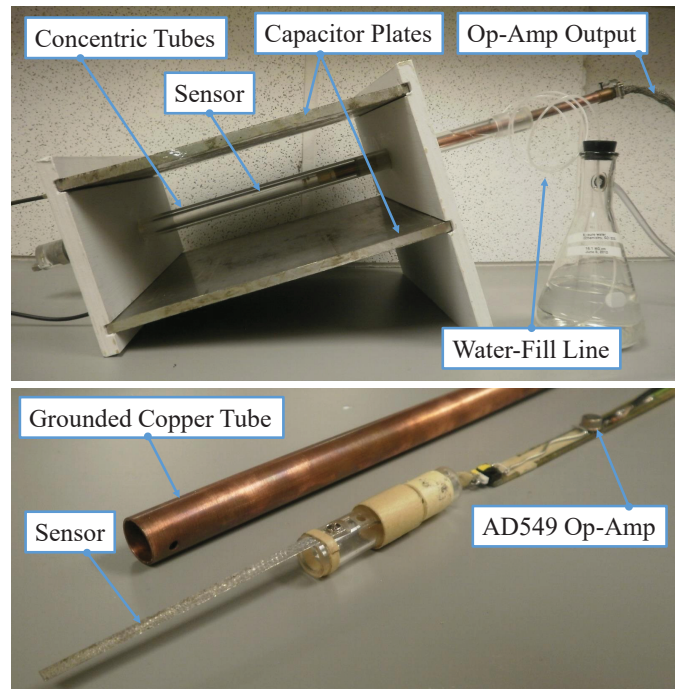

FIG. 3. Photographs of the apparatus. Top: Two concentric plexiglass tubes pass through the capacitor. The sensor is suspended within the inner tube. Bottom: The buffer circuit is contained within an electrically grounded copper tube. The copper tube fits inside the inner plexiglass tube and allows the sensor to be rotated into two symmetric positions.

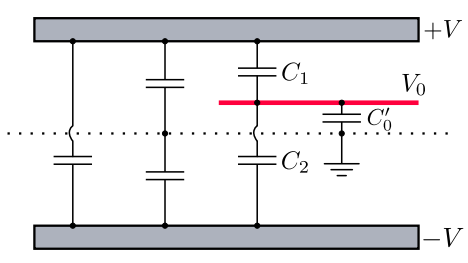

FIG. 4. A circuit model showing the electrode suspended just above the ground plane (dotted line) between oppositelycharged capacitor plates.

can occur between the electrode and the buffer input ${ }^{\infty}$. Many useful guidelines, such as tying the shield of the buffer input to the output, are given in Refs. $\square$ and $\square$.

As shown in Fig. [1, a pair of concentric plexiglass tubes are suspended midway between the capacitor plates. The outer tube has a inner diameter (id) of $1 \mathrm{in}$. and an outer diameter (od) of $1.25 \mathrm{in}$. The copper tube slides freely inside the inner tube (id $=5 / 8 \mathrm{in}$., od $=3 / 4 \mathrm{in}$.) and allows the electrode to be rotated into two symmetric positions. In this experiment, the space between the plexiglass tubes is filled with either air or water. The entire assembly is tilted at a slight angle so that, when the tubes are filled with water, there are no air pockets in the region between the capacitor plates.

A General Radio 1310-B function generator was used to drive the transformer which stepped down the voltage by a factor of eight at $1 \mathrm{kHz}$. The AD549L op-amp was powered using two $9 \mathrm{~V}$ batteries and its output was measured using a Princeton Applied Research 5204 lockin amplifier. The lock-in amplifier was referenced to the voltage applied to the top capacitor plate.

To probe the electric field within the inner plexiglass tube, and therefore test Eqs. $\mathbf{m}$ and $\mathbf{\square}$, the potentials at two symmetric positions were measured. The first position placed the electrode closer to the top plate as shown in Fig. \ and the second position placed it nearer to the bottom plate. The difference of these two readings is proportional to the electric field $E \propto V_{2}-V_{1}$ such that:

$$
\begin{aligned}
& E_{A} \cos (\omega t+\gamma) \propto \\
& \quad A_{2} \cos \left(\omega t+\theta_{2}\right)-A_{1} \cos \left(\omega t+\theta_{1}\right)
\end{aligned}
$$

where $E_{A}$ and $A$ are the amplitudes of the electric field and potentials respectively and $\gamma$ and $\theta$ are phases measured relative to the phase of the top-plate voltage. Application of some trigonometric identities leads to:

$$
\begin{aligned}
E_{A} & \propto \sqrt{A_{1}^{2}+A_{2}^{2}-2 A_{1} A_{2} \cos \Delta \theta} \\
\tan \gamma & =\frac{A_{2} \sin \theta_{2}-A_{1} \sin \theta_{1}}{A_{2} \cos \theta_{2}-A_{1} \cos \theta_{1}}
\end{aligned}
$$

where $\Delta \theta \equiv \theta_{2}-\theta_{1}$. As a result, $\gamma$ and a quantity proportional to the electric field amplitude can be extracted from the $V_{1}\left(A_{1}, \theta_{1}\right)$ and $V_{2}\left(A_{2}, \theta_{2}\right)$ measurements.

\section{EXPERIMENTAL RESULTS \& SUMMARY}

With air between the plexiglass tubes, the magnitude and phase of the electric field were measured as a function of frequency from $100 \mathrm{~Hz}$ to $300 \mathrm{kHz}$. This measurement was then repeated with water placed between the tubes. The water used was filtered by a Barnstead E-Pure water purification system. It is important to use high-purity water with a low conductivity so that the cutoff frequency given by $\sigma /\left(\varepsilon_{0} \varepsilon_{\mathrm{r}}\right)$ is as low as possible. The results are shown in Fig. 回. As expected, Figs. 回(a) and (b) show that the air measurement has a magnitude that is approximately flat and a phase that is close to zero at low frequencies. However, above $10 \mathrm{kHz}$ the magnitude and phase both start to decrease with increasing frequency. These effects are due to the high-frequency limitations of the op-amp and lock-in amplifier.

When the measurements are repeated with water between the tubes, the same high-frequency characteristics are observed, however, at low frequency there are differences. The magnitude of the electric field is very small at the lowest frequencies and the measured phase is approximately $\pi / 2$ radians. The frequency dependence of the detection electronics can be removed from the magnitude measurements by dividing the water data by the air data as shown in Fig. 0 (c). Because only a quantity that is proportional to the magnitude has been measured, the ratio of the water-to-air data have been scaled to approach one at high-frequency. The high-pass filter behaviour predicted by Eq. 四 is clearly exhibited. Likewise, the contribution of the detection electronics to the phase measurements can be removed by taking the difference of the water phase and the air phase. The tangent of the difference is shown in Fig. $\mathrm{g}(\mathrm{d})$ and exhibits the expected inverse-frequency behaviour of Eq. $\mathbf{\square}$. 

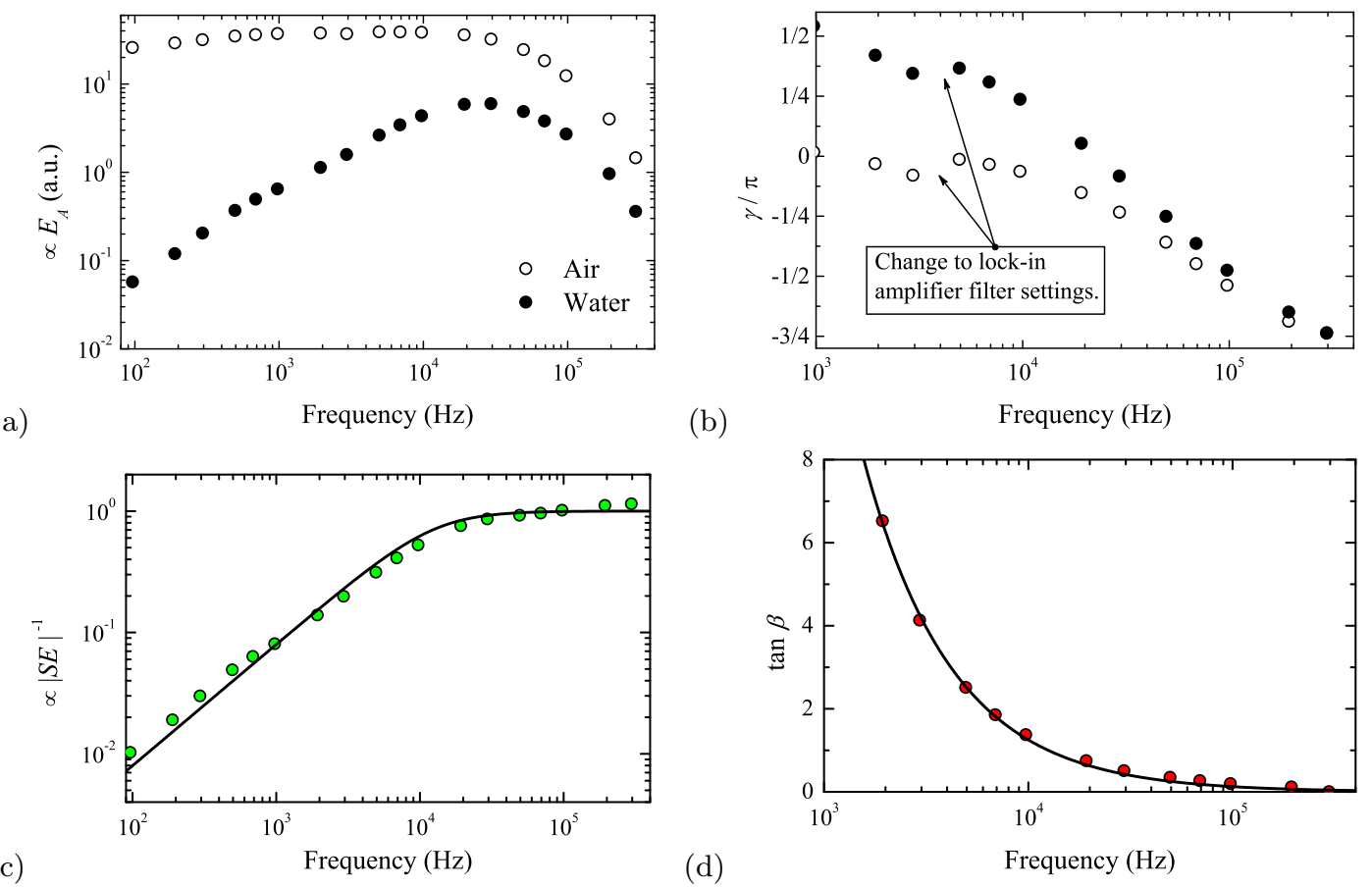

(b)

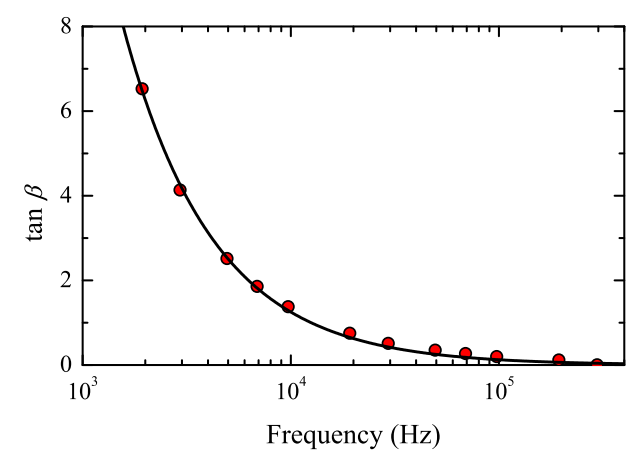

FIG. 5. (a) Frequency dependence of the electric field with air and water between the plexiglass tubes. (b) The corresponding phase measurements. (c) The frequency dependencies of the buffer and detection electronics are removed from the amplitude measurements by dividing the water data by the air data. The ratio was fit to Eq. $\mathbf{d}$. (d) The difference $\gamma_{\text {water }}-\gamma_{\text {air }}$ determines $\beta$. The $\tan \beta$ data were fit to Eq. $\square$. In all four plots, the measurement uncertainties are approximately equal to the size of the data points.

The $|S E|^{-1}$ and $\tan \beta$ data were simultaneously fit

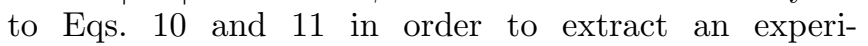
mental value for $\sigma /\left(\varepsilon_{0} \varepsilon_{\mathrm{r}}\right)=78700 \pm 500 \mathrm{~s}^{-1}$. For water at room temperature $\varepsilon_{\mathrm{r}}=79 \pm 1^{\mathbb{\boxplus}}$, such that $\sigma=0.550 \pm 0.008 \mathrm{M} \Omega^{-1} \mathrm{~cm}^{-1}$ which is a reasonable value for the E-pure water used in the experiment.

An experiment to measure the frequency dependence and phase of the shielding effectiveness of a tube of water has been designed, built, and executed. It is a novel experiment for senior physics undergraduates that is rich in both theoretical physics and measurement techniques.
Investigating the screening of electric fields by a dielectric tube is a standard exercise in electrodynamics ${ }^{\mathbf{W}}$ and including the conductivity of water is a unique extension of the problem. Students are challenged to develop a circuit model for an electrode suspended between capacitor plates and hence understand why a high-impedance buffer is required. Students are also introduced to the powerful technique of lock-in detection. The experimental results clearly show the predicted high-pass filter frequency dependence of the shielding effectiveness and reasonable values for the ratio of the water's conductivity to its dielectric constant are extracted.
* Take.Bobowskiaubc.ca; https://people.ok.ubc.ca/jbobowsk

$\dagger$ Also at UrtheCast, 1055 Canada Place - Suite 33, Vancouver, BC V6C 0C3, Canada

${ }^{1}$ S. Celozzi, R. Araneo, and G. Lovat, Electromagnetic Shielding (John Wiley \& Sons, 2008).

2 D. J. Griffiths, Introduction to Electrodynamics, 3rd ed. (Prentice Hall, 1999).

3 J. D. Jackson, Classical Electrodynamics, 3rd ed. (John Wiley \& Sons, 1998).

${ }^{4}$ C. G. Malmberg and A. A. Maryott, J. Res. Natl. Stand. 56, 2641 (1956).

5 J. S. Bobowski, T. Johnson, and C. Eskicioglu, Prog. Electromagn. Res. Lett. 29, 139-149 (2012).

${ }^{6}$ J. S. Bobowski and T. Johnson, Prog. Electromagn. Res.
B 40, 159-183 (2012).

7 J. S. Bobowski, Am. J. Phys. 81, 899-906 (2013).

8 The four-boundary problem was solved using the mathematical analysis software Maple 18 and then verified using the finite-element simulation tool COMSOL 4.3b.

9 J. De Vos, B.Sc. thesis, University of British Columbia (2014).

10 J. S. Bobowski, Md. S. Ferdous, and T. Johnson, IEEE Trans. Instrum. Meas. 64, 923-934 (2015).

11 AD549 Datasheet: Ultralow input bias current operational amplifier, Analog Devices, Inc. (2008).

12 AD515A Datasheet: Monolithic precision, low power FETinput electrometer op amp, Analog Devices, Inc. (1997). 